\title{
Stability and Boundedness of Solutions to a Certain Second-Order Nonautonomous Stochastic Differential Equation
}

\author{
A. T. Ademola, ${ }^{1}$ S. Moyo, ${ }^{2}$ B. S. Ogundare, ${ }^{1}$ M. O. Ogundiran, ${ }^{1}$ and O. A. Adesina ${ }^{1}$ \\ ${ }^{1}$ Research Group in Differential Equations and Applications (RGDEA), Department of Mathematics, Obafemi Awolowo University, \\ Ile-Ife 220005, Nigeria \\ ${ }^{2}$ Institute for Systems Science \& Research and Postgraduate Support Directorate, Durban University of Technology, \\ Durban 4000, South Africa
}

Correspondence should be addressed to O. A. Adesina; oadesina@oauife.edu.ng

Received 28 July 2016; Revised 9 November 2016; Accepted 27 November 2016

Academic Editor: Ying $\mathrm{Hu}$

Copyright (C) 2016 A. T. Ademola et al. This is an open access article distributed under the Creative Commons Attribution License, which permits unrestricted use, distribution, and reproduction in any medium, provided the original work is properly cited.

This paper focuses on stability and boundedness of certain nonlinear nonautonomous second-order stochastic differential equations. Lyapunov's second method is employed by constructing a suitable complete Lyapunov function and is used to obtain criteria, on the nonlinear functions, that guarantee stability and boundedness of solutions. Our results are new; in fact, according to our observations from the relevant literature, this is the first attempt on stability and boundedness of solutions of second-order nonlinear nonautonomous stochastic differential equations. Finally, examples together with their numerical simulations are given to authenticate and affirm the correctness of the obtained results.

\section{Introduction}

Differential equations of second-order have generated a great deal of applications in various fields of science and technology such as biology, chemistry, physics, mechanics, control technology, communication network, automatic regulation, economy, and ecology to mention few. In addition, the study of problems that involve the behaviour of solutions of ordinary differential equations (ODE), delay or functional differential equations (DDE), and stochastic differential equations (SDE) has been dealt with by many outstanding authors; see, for instance, Arnold [1], Burton [2, 3], Hale [4], Oksendal [5], Shaikihet [6], and Yoshizawa [7, 8], which contain the background to the study and the expository papers of Abou-El-Ela et al. [9, 10], Ademola et al. [11, 12], Alaba and Ogundare [13], Burton and Hatvani [14], Cahlon and Schmidt [15], Caraballo et al. [16], Domoshnitsky [17], Gikhman and Skorokhod [18, 19], Grigoryan [20], Ivanov et al. [21], Jedrzejewski and Brochard [22], Jin and Zengrong [23], Kolarova [24], Kolmanovskii and Shaikhet [25, 26], Kroopnick [27], Liu and Raffoul [28], Mao [29], Ogundare et al. [30-32], Raffoul [33], Rezaeyan and Farnoosh [34], Tunç [35-43], Wang and Zhu [44], Xianfeng and Wei [45],

Yeniçerioğlu [46, 47], Yoshizawa [48], Zhu et al. [49], and the references cited therein.

The authors in $[18,19]$ investigated the second-order linear scalar equations of the form

$$
Y_{t}^{\prime \prime}+\left(a(t)+b(t) \eta_{i}\right) \dot{Y}_{t}=0, \quad t \geq t_{0}
$$

where $\dot{\eta}_{i}$ is a general disturbance process (the derivative of a martingale). In [11, 12] the authors discussed stability, boundedness, and periodic solutions to the following secondorder ordinary and delay differential equations:

$$
\begin{aligned}
& {\left[\phi(x(t)) x^{\prime}(t)\right]^{\prime}+g\left(t, x(t), x^{\prime}(t)\right) x^{\prime}(t)} \\
& \quad+\varphi(t) h(x(t))=p\left(t, x(t), x^{\prime}(t)\right), \\
& x^{\prime \prime}(t) \\
& \quad+\phi(t) f\left(x(t), x(t-\tau(t)), x^{\prime}(t), x^{\prime}(t-\tau(t))\right) \\
& \quad+g(x(t-\tau(t)))=p\left(t, x(t), x^{\prime}(t)\right),
\end{aligned}
$$


respectively, where $f, g, p, h, \phi$, and $\varphi$ are continuous functions in their respective arguments. In their contributions, the authors in $[9,10]$ investigated asymptotic stability and boundedness of solutions of the following second-order stochastic delay differential equations:

$$
\begin{aligned}
& x^{\prime \prime}(t)+a x^{\prime}(t)+b x(t-h)+\sigma x(t) \omega^{\prime}(t)=0, \\
& x^{\prime \prime}(t)+a x^{\prime}(t)+f(x(t-h))+\sigma x(t-\tau) \omega^{\prime}(t)=0, \\
& x^{\prime \prime}(t)+g\left(x^{\prime}(t)\right)+b x(t-h)+\sigma x(t) \omega^{\prime}(t) \\
& \quad=p\left(t, x(t), x^{\prime}(t), x^{\prime}(t-h)\right),
\end{aligned}
$$

respectively, where $a, b$, and $\sigma$ are positive constants; $h, \tau$ are delay constants; $f, g$, and $p$ are continuous functions in their respective arguments and $w(t) \in \mathbb{R}^{m}$ is an $m$-dimensional standard Brownian motion defined on the probability space (also called Wiener process). Recently, in 2016 the authors in [43] discussed global existence and boundedness of solutions of a certain nonlinear integrodifferential equation of secondorder with multiple deviating arguments

$$
\begin{aligned}
& {\left[p(x(t)) x^{\prime}(t)\right]^{\prime}+a(t) f\left(t, x(t), x^{\prime}(t)\right) x^{\prime}(t)} \\
& \quad+b(t) g\left(t, x^{\prime}(t)\right)+\sum_{i=1}^{n} c_{i}(t) h_{i}\left(x\left(t-\tau_{i}\right)\right) \\
& =\int_{0}^{t} c(t, s) x^{\prime}(s) d s,
\end{aligned}
$$

where $\tau_{i}(i=1,2, \ldots, n)$ are positive constants, $a, b$, and $c$ are defined on $\mathbb{R}^{+}$, and $f, g, h$, and $p$ are continuous functions defined in their respective arguments.

Although second-order stochastic delay differential equations have started receiving attention of authors, according to our observation from relevant literature, there is no previous literature available on the stability and boundedness of solutions of second-order nonlinear nonautonomous stochastic differential equation. The aim of this paper is to bridge this gap. Consider the following second-order nonlinear nonautonomous stochastic differential equation:

$$
\begin{aligned}
x^{\prime \prime}(t) & +g\left(x(t), x^{\prime}(t)\right) x^{\prime}(t)+f(x(t)) \\
& +\sigma x(t) \omega^{\prime}(t)=p\left(t, x(t), x^{\prime}(t)\right),
\end{aligned}
$$

where $\sigma$ is a positive constant, the functions $g, f$, and $p$ are continuous in their respective arguments on $\mathbb{R}^{2}, \mathbb{R}$, and $\mathbb{R}^{+} \times \mathbb{R}^{2}$, respectively, with $\mathbb{R}:=(-\infty, \infty), \mathbb{R}^{+}:=[0, \infty)$, and $\omega$ (a standard Wiener process, representing the noise) is defined on $\mathbb{R}$. Furthermore, it is assumed that the continuity of the functions $g, f$, and $p$ is sufficient for the existence of solutions and the local Lipschitz condition for (8) to have a unique continuous solution denoted by $(x(t), y(t))$. The primes denote differentiation with respect to the independent variable $t \in \mathbb{R}^{+}$. If $x^{\prime}(t)=y(t)$, then (8) is equivalent to the system:

$$
\begin{aligned}
x^{\prime}(t)= & y(t) \\
y^{\prime}(t)= & p(t, x(t), y(t))-f(x)-g(x(t), y(t)) y(t) \\
& -\sigma x(t) \omega^{\prime}(t)
\end{aligned}
$$

where the derivative of the function $f$ (i.e., $f^{\prime}$ ) exists and is continuous for all $x$. Despite the applicability of these classes of equations, there is no previous result on nonautonomous second-order nonlinear stochastic differential equation (8). The motivation for this investigation comes from the works in $[9-12,18,19]$. If $\sigma=0$ in (8), then we have a general second-order nonlinear ordinary differential equation which has been discussed extensively in relevant literature. The remaining parts of this paper are organized as follows. In Section 2, we give the preliminary results on stochastic differential equations. Main results and their proofs are presented in Section 3 while examples and simulation of solutions are given in Section 4 to validate our results.

\section{Preliminary Results}

Let $\left(\Omega, \mathfrak{F},\left\{\mathfrak{F}_{t}\right\}_{t>0}, \mathbb{P}\right)$ be a complete probability space with a filtration $\left\{\mathfrak{F}_{t}\right\}_{t>0}$ satisfying the usual conditions (i.e., it is right continuous and $\left\{\mathfrak{F}_{0}\right\}$ contains all $\mathbb{P}$-null sets). Let $B(t)=$ $\left(B_{1}(t), \ldots, B_{m}(t)\right)^{T}$ be an $m$-dimensional Brownian motion defined on the probability space. Let $|\cdot|$ denotes the Euclidean norm in $\mathbb{R}^{n}$. If $A$ is a vector or matrix, its transpose is denoted by $A^{T}$. If $A$ is a matrix, its trace norm is denoted by

$$
|A|=\sqrt{\operatorname{trace}\left(A^{T} A\right)} .
$$

For more exposition in this regard, see Mao [29] and Arnold [1]. Now let us consider a nonautonomous $n$-dimensional stochastic differential equation

$$
d X(t)=F(t, X(t)) d t+G(t, X(t)) d B(t)
$$

on $t>0$ with initial value $X(0)=X_{0} \in \mathbb{R}^{n}$. Here $F: \mathbb{R}^{+} \times$ $\mathbb{R}^{n} \rightarrow \mathbb{R}^{n}$ and $G: \mathbb{R}^{+} \times \mathbb{R}^{n} \rightarrow \mathbb{R}^{n \times m}$ are measurable functions. Suppose that both $F$ and $G$ are sufficiently smooth for (11) to have a unique continuous solution on $t \geq 0$ which is denoted by $X\left(t, X_{0}\right)$, if $\mathrm{X}(0)=0$. Assume further that

$$
F(t, 0)=G(t, 0)=0
$$

for all $t \geq 0$. Then, the stochastic differential equation (11) admits zero solution $X(t, 0) \equiv 0$.

Definition 1 (see [1]). The zero solution of the stochastic differential equation (11) is said to be stochastically stable or stable in probability, if for every pair of $\epsilon \in(0,1)$ and $r>0$, there exists a $\delta_{0}=\delta_{0}(\epsilon, r)>0$ such that

$$
\begin{aligned}
\operatorname{Pr}\left\{\left|X\left(t ; X_{0}\right)\right|<r \forall t \geq 0\right\} \geq 1-\epsilon & \\
& \text { whenever }\left|X_{0}\right|<\delta_{0} .
\end{aligned}
$$

Otherwise, it is said to be stochastically unstable. 
Definition 2 (see [1]). The zero solution of the stochastic differential equation (11) is said to be stochastically asymptotically stable if it is stochastically stable and in addition if for every $\epsilon \epsilon(0,1)$ and $r>0$, there exists a $\delta=\delta(\epsilon)>0$ such that

$$
\operatorname{Pr}\left\{\lim _{t \rightarrow \infty} X\left(t ; X_{0}\right)=0\right\} \geq 1-\epsilon \quad \text { whenever }\left|X_{0}\right|<\delta .
$$

Definition 3. A solution $X\left(t_{0}, X_{0}\right)$ of the stochastic differential equation (11) is said to be stochastically bounded or bounded in probability, if it satisfies

$$
E^{X_{0}}\left\|X\left(t, X_{0}\right)\right\| \leq C\left(t_{0},\left\|X_{0}\right\|\right), \quad \forall t \geq t_{0}
$$

where $E^{X_{0}}$ denotes the expectation operator with respect to the probability law associated with $X_{0}, C: \mathbb{R}^{+} \times \mathbb{R}^{n}$ and $\mathbb{R}^{+}$is a constant depending on $t_{0}$ and $X_{0}$.

Definition 4. The solutions $X\left(t_{0}, X_{0}\right)$ of the stochastic differential equation (11) are said to be uniformly stochastically bounded if $C$ in inequality (15) is independent of $t_{0}$.

For $h>0$, let $U_{h}=\left\{X \in \mathfrak{R}^{n}:|X|<h\right\} \subset \mathbb{R}^{n}$ and let $C^{1,2}\left(U_{h} \times \mathbb{R}^{+}, \mathbb{R}^{+}\right)$denote the family of all nonnegative functions $V(t, X(t))$ (Lyapunov function) defined on $\mathbb{R}^{+} \times U_{h}$ which are twice continuously differentiable in $X$ and once in $t$. By Itô's formula we have

$$
\begin{aligned}
d V(t, X(t))= & L V(t, X(t)) d t \\
& +V_{x}(t, X(t)) G(t, X(t)) d B(t),
\end{aligned}
$$

where

$$
\begin{aligned}
L V(t, X(t)) & \\
= & \frac{\partial V(t, X(t))}{\partial t}+\frac{\partial V(t, X(t))}{\partial x_{i}} F(t, X(t)) \\
& +\frac{1}{2} \operatorname{trace}\left[G^{T}(t, X(t)) V_{x x}(t, X(t)) G(t, X(t))\right] .
\end{aligned}
$$

Furthermore,

$$
V_{x x}(t, X(t))=\left(\frac{\partial^{2} V(t, X(t))}{\partial x_{i} \partial x_{j}}\right)_{n \times n}, \quad i, j=1, \ldots, n .
$$

In this study we will use the diffusion operator $L V(t, X(t))$ defined in (17) to replace $V^{\prime}(t, X(t))=(d / d t) V(t, X(t))$. We now present the basic results that will be used in the proofs of the main results.

Lemma 5 (see [1]). Assume that there exist $V \in C^{1,2}\left(\mathbb{R}^{+} \times\right.$ $\left.U_{h}, \mathbb{R}^{+}\right)$and $\phi \in \mathbb{K}$ such that

(i) $V(t, 0)=0$;

(ii) $V(t, X(t))>\phi(\|X(t)\|)$;

(iii) $L V(t, X(t)) \leq 0$ for all $(t, X) \in \mathbb{R}^{+} \times U_{h}$.

Then the zero solution of stochastic differential equation (11) is stochastically stable.
Lemma 6 (see [1]). Suppose that there exist $V \in C^{1,2}\left(\mathbb{R}^{+} \times\right.$ $\left.U_{h}, \mathbb{R}^{+}\right)$and $\phi_{0}, \phi_{1}, \phi_{2} \in \mathbb{K}$ such that

(i) $V(t, 0)=0$;

(ii) $\phi_{0}(\|X(t)\|) \leq V(t, X(t)) \leq \phi_{1}(\|X(t)\|), \phi_{0}(r) \rightarrow \infty$ as $r \rightarrow \infty$;

(iii) $L V(t, X(t)) \leq-\phi_{2}(\|X(t)\|)$ for all $(t, X) \in \mathbb{R}^{+} \times U_{h}$.

Then the zero solution of stochastic differential equation (11) is uniformly stochastically asymptotically stable in the large.

Assumption 7 (see $[28,33])$. Let $V \in C^{1,2}\left(\mathbb{R}^{+} \times \mathbb{R}^{n} ; \mathbb{R}^{+}\right)$, and suppose that for any solutions $X\left(t_{0}, X_{0}\right)$ of stochastic differential equation (11) and for any fixed $0 \leq t_{0} \leq T<\infty$, we have

$$
\begin{aligned}
E^{X_{0}}\left\{\int_{t_{0}}^{T} V_{x_{i}}^{2}(t, X(t)) G_{i k}^{2}(t, X(t)) d t\right\} & <\infty, \\
& 1 \leq i \leq n, 1 \leq k \leq m .
\end{aligned}
$$

Assumption 8 (see [28, 33]). A special case of the general condition (19) is the following condition. Assume that there exits a function $\sigma(t)$ such that

$$
\begin{aligned}
\left|V_{x_{i}}(t, X(t)) G_{i k}(t, X(t))\right| & <\sigma(t), \\
X & \in \mathbb{R}^{n} 1 \leq i \leq n, 1 \leq k \leq m,
\end{aligned}
$$

and for any fixed $0 \leq t_{0} \leq T<\infty$,

$$
\int_{t_{0}}^{T} \sigma^{2}(t) d t<\infty
$$

Lemma 9 (see $[28,33])$. Assume there exists a Lyapunov function $V(t, X(t)) \in C^{1,2}\left(\mathbb{R}^{+} \times \mathbb{R}^{n} ; \mathbb{R}^{+}\right)$, satisfying Assumption 7 , such that, for all $(t, X) \in \mathbb{R}^{+} \times \mathbb{R}^{n}$,

(i) $\|X(t)\|^{p} \leq V(t, X(t)) \leq\|X(t)\|^{q}$,

(ii) $L V(t, X(t)) \leq-\alpha(t)\|X(t)\|^{r}+\beta(t)$,

(iii) $V(t, X(t))-V^{r / q}(t, X(t)) \leq \gamma$,

where $\alpha, \beta \in C\left(\mathbb{R}^{+} ; \mathbb{R}^{+}\right), p$, $q$, and $r$ are positive constants, $p \geq 1$, and $\gamma$ is a nonnegative constant. Then all solutions of the stochastic differential equation (11) satisfy

$$
\begin{gathered}
E^{X_{0}}\left\|X\left(t, X_{0}\right)\right\| \leq\left\{V\left(t_{0}, X_{0}\right) e^{-\int_{t_{0}}^{t} \alpha(s) d s}\right. \\
\left.+\int_{t_{0}}^{t}(\gamma \alpha(u)+\beta(u)) e^{-\int_{u}^{t} \alpha(s) d s} d u\right\}^{1 / p},
\end{gathered}
$$

for all $t \geq t_{0}$.

Lemma 10 (see $[28,33])$. Assume there exists a Lyapunov function $V(t, X(t)) \in C^{1,2}\left(\mathbb{R}^{+} \times \mathbb{R}^{n} ; \mathbb{R}^{+}\right)$, satisfying Assumption 7 , such that, for all $(t, X) \in \mathbb{R}^{+} \times \mathbb{R}^{n}$,

(i) $\|X(t)\|^{p} \leq V(t, X(t))$,

(ii) $L V(t, X(t)) \leq-\alpha(t) V^{q}(t, X(t))+\beta(t)$,

(iii) $V(t, X(t))-V^{q}(t, X(t)) \leq \gamma$, 
where $\alpha, \beta \in C\left(\mathbb{R}^{+} ; \mathbb{R}^{+}\right), p$, q are positive constants, $p \geq 1$, and $\gamma$ is a nonnegative constant. Then all solutions of the stochastic differential equation (11) satisfy (22) for all $t \geq t_{0}$.

Corollary 11 (see [28, 33]). (i) Assume that hypotheses (i) to (iii) of Lemma 9 hold. In addition,

$$
\int_{t_{0}}^{t}(\gamma \alpha(u)+\beta(u)) e^{-\int_{u}^{t} \alpha(s) d s} d u \leq M, \quad \forall t \geq t_{0} \geq 0,
$$

for some positive constant $M$; then all solutions of stochastic differential equation (11) are uniformly stochastically bounded.

(ii) Assume that hypotheses (i) to (iii) of Lemma 10 hold. If condition (23) is satisfied, then all solutions of the stochastic differential equation (11) are stochastically bounded.

\section{Main Results}

Let $(x(t), y(t))$ be any solution of the stochastic differential equation (9); the main tool employed in the proofs of our results is the continuously differentiable function $V=$ $V(t, x(t), y(t))$ defined as

$$
2 V=b^{2} x^{2}+b y^{2}+2 x f(x)+(a x+y)^{2},
$$

where $a$ and $b$ are positive constants and the function $f$ is as defined in Section 1.

Theorem 12. Suppose that $a, b, \sigma$, and $M_{0}$ are positive constants such that

(i) $a \leq g(x, y)$ for all $x$ and $y$,

(ii) $b x \leq f(x) \leq B x$ for all $x \neq 0$ and $\sigma^{2}<2 a b(b+1)^{-1}$,

(iii) $|p(t, x, y)| \leq M_{0}$ for all $t \geq 0, x$ and $y$.

Then solution $(x(t), y(t))$ of the stochastic differential equation (9) is uniformly stochastically bounded.

Remark 13. We note the following:

(i) Whenever the functions $g\left(x, x^{\prime}\right)=a, f(x)=b x$ and $\omega^{\prime}=p\left(t, x, x^{\prime}\right)=0$, then the stochastic differential equation (8) becomes a second-order linear ordinary differential equation

$$
x^{\prime \prime}+a x^{\prime}+b x=0,
$$

and conditions (i) to (iii) of Theorem 12 reduce to Routh Hurwitz criteria $a>0$ and $b>0$ for the asymptotic stability of the second-order linear differential equation (25).

(ii) The term $\sigma x(t) \omega^{\prime}(t)$ in the stochastic differential equation (8) is an extension of the ordinary case discussed recently by authors in $[11,18,23,31,32,35-$ 37, 40].

We shall now state and prove a result that will be used in the proofs of our results.
Lemma 14. Under the hypotheses of Theorem 12, there exist positive constants $D_{0}=D_{0}(a, b)$ and $D_{1}=D_{1}(a, b, B)$ such that

$$
\begin{aligned}
D_{0}\left(x^{2}(t)+y^{2}(t)\right) & \leq V(t, x(t), y(t)) \\
& \leq D_{1}\left(x^{2}(t)+y^{2}(t)\right),
\end{aligned}
$$

for all $t \geq 0, x$, and $y$. In addition, there exist positive constants $D_{2}=D_{2}(a, b, \sigma)$ and $D_{3}=D_{3}(a, b)$ such that

$$
\begin{aligned}
L V(t, x(t), y(t)) & \\
\leq & -D_{2}\left(x^{2}(t)+y^{2}(t)\right) \\
& +D_{3}(|x(t)|+|y(t)|)|p(t, x(t), y(t))|,
\end{aligned}
$$

for all $t \geq 0, x$, and $y$.

Proof. Let $(x(t), y(t))$ be any solution of the stochastic differential equation (9); since $X=(x, y) \in \mathbb{R}^{2}$, it follows from (24) that

$$
V(t, 0,0)=0,
$$

for all $t \geq 0$. Moreover, from (24) and the fact that $f(x) \geq a x$ for all $x \neq 0$, there exists a positive constant $\delta_{0}$ such that

$$
V(t, X) \geq \delta_{0}\left(x^{2}+y^{2}\right)
$$

for all $t \geq 0, x$, and $y$, where

$$
\delta_{0}:=\min \left\{b^{2}+2 b+\min \{a, 1\}, b+\min \{a, 1\}\right\} .
$$

It is clear from inequality (29) that

$$
\begin{aligned}
& V(t, X)=0 \Longleftrightarrow x^{2}+y^{2}=0, \\
& V(t, X)>0 \Longleftrightarrow x^{2}+y^{2} \neq 0, \\
& V(t, X) \longrightarrow+\infty \quad \text { as } x^{2}+y^{2} \longrightarrow \infty .
\end{aligned}
$$

Furthermore, since $f(x) \leq B x$ for all $x \neq 0$, it follows from (24) that there exists a positive constant $\delta_{1}$ such that

$$
V(t, X) \leq \delta_{1}\left(x^{2}+y^{2}\right)
$$

for all $t \geq 0, x$, and $y$, where

$$
\delta_{1}:=\max \left\{b^{2}+2 B+\max \{a, 1\}, b+\max \{a, 1\}\right\} .
$$

From inequalities (29) and (33), we have

$$
\delta_{0}\left(x^{2}+y^{2}\right) \leq V(t, X) \leq \delta_{1}\left(x^{2}+y^{2}\right),
$$

for all $t \geq 0, x$, and $y$. It is not difficult to see that estimates (35) satisfy inequalities (26) of Lemma 14 with $\delta_{0}$ and $\delta_{1}$ equivalent to $D_{0}$ and $D_{1}$, respectively. 
Moreover, applying Itô's formula in (24) using system (9), we find that

$$
\begin{aligned}
L V(t, X)= & \frac{1}{2}\left[a \frac{f(x)}{x}-\frac{1}{2} \sigma^{2}(b+1)\right] x^{2} \\
& -\frac{1}{2}[(b+1) g(x, y)-a] y^{2}-W_{i} \\
& +[a x+(b+1) y] p(t, x, y),
\end{aligned}
$$

$$
(i=1,2)
$$

where

$$
\begin{aligned}
W_{1} & :=\frac{1}{4}\left\{\left[a \frac{f(x)}{x}-\frac{1}{2} \sigma^{2}(b+1)\right] x^{2}\right. \\
+ & 4\left[a g(x, y)-\left(a^{2}+b^{2}\right)\right] x y \\
& \left.+[(b+1) g(x, y)-a] y^{2}\right\}, \\
W_{2} & :=\frac{1}{4}\left\{\left[a \frac{f(x)}{x}-\frac{1}{2} \sigma^{2}(b+1)\right] x^{2}\right. \\
& +4\left[a \frac{f(x)}{x}-f^{\prime}(x)\right] x y \\
& \left.+[(b+1) g(x, y)-a] y^{2}\right\} .
\end{aligned}
$$

It is clear from the inequalities

$$
\begin{aligned}
& 4\left[a g(x, y)-\left(a^{2}+b^{2}\right)\right]^{2} \\
& \quad<\left[a \frac{f(x)}{x}-\frac{1}{2} \sigma^{2}(b+1)\right][(b+1) g(x, y)-a], \\
& 4\left[a \frac{f(x)}{x}-f^{\prime}(x)\right] \\
& \quad<\left[a \frac{f(x)}{x}-\frac{1}{2} \sigma^{2}(b+1)\right][(b+1) g(x, y)-a]
\end{aligned}
$$

that

$$
\begin{gathered}
W_{1}=W_{2} \geq\left[\sqrt{a \frac{f(x)}{x}-\frac{1}{2} \sigma^{2}(b+1)}|x|\right. \\
-\sqrt{(b+1) g(x, y)-a}|y|]^{2} \geq 0,
\end{gathered}
$$

for all $x$ and $y$. Using inequality (39) and hypotheses (i) and (ii) of Theorem 12 in (36), there exist positive constants $\delta_{2}$ and $\delta_{3}$ such that

$$
\begin{aligned}
L V(t, X) \leq & -\delta_{2}\left(x^{2}+y^{2}\right) \\
& +\delta_{3}(|x|+|y|)|p(t, x, y)|
\end{aligned}
$$

for all $t \geq 0, x$, and $y$, where

$$
\begin{aligned}
\delta_{2} & :=\frac{1}{2} \min \left\{a b-\frac{1}{2} \sigma^{2}(b+1), a b\right\}, \\
\delta_{3} & :=\max \{a, b+1\} .
\end{aligned}
$$

Inequality (40) satisfies inequality (27) with $\delta_{2}$ and $\delta_{3}$ equivalent to $D_{2}$ and $D_{3}$, respectively. This completes the proof of Lemma 14.

Proof of Theorem 12. Let $(x(t), y(t))$ be any solution of system (9). From inequality (40) and assumption (iii) of Theorem 12, we have

$$
\begin{aligned}
& L V(t, X) \\
& \begin{aligned}
\leq & -\frac{1}{2} \delta_{2}\left(x^{2}+y^{2}\right) \\
& -\frac{1}{2} \delta_{2} M_{0}\left[\left(|x|-\delta_{2}^{-1} \delta_{3}\right)^{2}+\left(|y|-\delta_{2}^{-1} \delta_{3}\right)^{2}\right] \\
& +M_{0} \delta_{2}^{-1} \delta_{3}^{2},
\end{aligned}
\end{aligned}
$$

for $t \geq 0, x$, and $y$. Since $\delta_{2}, \delta_{3}$, and $M_{0}$ are positives and

$$
\left(|x|-\delta_{2}^{-1} \delta_{3}\right)^{2}+\left(|y|-\delta_{2}^{-1} \delta_{3}\right)^{2} \geq 0,
$$

for all $x$ and $y$, there exist positive constants $\delta_{4}$ and $\delta_{5}$ such that

$$
L V(t, X) \leq-\delta_{4}\left(x^{2}+y^{2}\right)+\delta_{5}
$$

for all $t \geq 0, x, y$, where $\delta_{4}:=(1 / 2) \delta_{2}$ and $\delta_{5}:=M_{0} \delta_{2}^{-1} \delta_{3}^{2}$. Hence, condition (ii) of Lemma 9 is satisfied with $\alpha(t):=\delta_{4}$, $r:=2$ and $\beta(t):=\delta_{5}$. Also from inequality (35), hypotheses (i) and (iii) of Lemma 9 hold with $p=q=2$ so that $\gamma=0$.

Furthermore, from inequality (23) we have

$$
\begin{aligned}
& \int_{t_{0}}^{t}\left[(\gamma \alpha(u)+\beta(u)) e^{-\delta_{4} \int_{u}^{t} \alpha(s) d s}\right] d u \\
& \quad=\int_{t_{0}}^{t} \delta_{5} e^{-\delta_{4} \int_{u}^{t} d s} d u=\delta_{4}^{-1} \delta_{5}\left[1-e^{-\delta_{4}\left(t-t_{0}\right)}\right] \\
& \quad \leq \delta_{4}^{-1} \delta_{5},
\end{aligned}
$$

for all $t \geq t_{0} \geq 0$. Inequality (45) satisfies estimate (23) with $M:=\delta_{4}^{-1} \delta_{5}=2 M_{0} \delta_{2}^{-2} \delta_{3}^{2}>0$. Moreover, from (9) and (24) there exists a positive constant $\delta_{6}$ such that

$$
\begin{aligned}
& \left|V_{x_{i}}(t, X) G_{i k}(t, X)\right| \\
& \quad \leq \frac{1}{2} \sigma\left[(2 a+b+1) x^{2}+(b+1) y^{2}\right] \\
& \quad \leq \delta_{6}\left(x^{2}+y^{2}\right):=\lambda(t),
\end{aligned}
$$

where

$$
\delta_{6}:=\frac{1}{2} \sigma \max \{2 a+b+1, b+1\} .
$$


Also,

$$
\int_{t_{0}}^{T} \delta_{6}^{2}\left(x^{2}(t)+y^{2}(t)\right)^{2} d t<\infty
$$

for any fixed $0 \leq t_{0} \leq T<\infty$. Thus, from inequalities (46) and (48) estimates (20) and (21) hold, respectively. Finally, from inequalities (33) and (45), we have

$$
E^{X_{0}}\left\|X\left(t, X_{0}\right)\right\| \leq\left(\delta_{1} X_{0}^{2}+2 M_{0} \delta_{2}^{-2} \delta_{3}^{2}\right)^{1 / 2},
$$

for all $t \geq t_{0} \geq 0$, where $X_{0}:=\left(x_{0}^{2}+y_{0}^{2}\right)$ and $C:=\delta_{1}$. Thus, the solutions $(x(t), y(t))$ of the stochastic differential equation (9) are uniformly stochastically bounded.

Theorem 15. If assumptions of Theorem 12 hold, then the solution $(x(t), y(t))$ of the stochastic differential equation (9) is stochastically bounded.

Proof. Suppose that $(x(t), y(t))$ is any solution of the stochastic differential equation (9). From inequalities (33) and (44) there exists a positive constant $\delta_{7}$ such that

$$
L V(t, X) \leq-\delta_{7} V(t, X)+\delta_{5}
$$

for all $t \geq 0, x$, and $y$, where $\delta_{7}:=\delta_{1}^{-1} \delta_{4}$. Hence, from inequalities (29) and (50) hypotheses of Lemma 10 hold. Moreover, from inequalities (45), (46), (48), and (49) assumption (ii) of Corollary 11 holds. Thus, by Corollary 11, all solutions of the stochastic differential equation (9) are stochastically bounded. This completes the proof of Theorem 15 .

Next, we shall discuss the stability of the trivial solution of the stochastic differential equation (8). Suppose that $p\left(t, x, x^{\prime}\right)=0,(8)$ specializes to

$$
\begin{aligned}
x^{\prime \prime}(t) & +g\left(x(t), x^{\prime}(t)\right) x^{\prime}(t)+f(x(t)) \\
& +\sigma x(t) \omega^{\prime}(t)=0 .
\end{aligned}
$$

Equation (51) has the following equivalent system:

$$
\begin{aligned}
& x^{\prime}(t)=y(t) \\
& y^{\prime}(t)=-f(x)-g(x(t), y(t)) y(t)-\sigma x(t) \omega^{\prime}(t),
\end{aligned}
$$

where the functions $f, g$, and $\omega$ are defined in Section 1 .

Theorem 16. If assumptions (i) and (ii) of Theorem 12 hold, then the trivial solution of the stochastic differential equation (52) is stochastically stable.

Proof. Let $(x(t), y(t))$ be any solution of the stochastic differential equation (52). From equation (28) and estimate (29) assumptions (i) and (ii) of Lemma 5 hold so that the function $V(t, X)$ is positive definite. Furthermore, using Itô's formula along the solution path of (52), we obtain

$$
L V(t, X) \leq-\delta_{2}\left(x^{2}(t)+y^{2}(t)\right) \leq 0,
$$

for all $t \geq 0, x$, and $y$, where $\delta_{2}$ is defined in (40). Inequality (53) satisfies hypothesis (iii) of Lemma 5; hence, by Lemma 5 the trivial solution of the stochastic differential equation (52) is stochastically stable. This completes the proof of Theorem 16.

Theorem 17. If assumptions (i) and (ii) of Theorem 12 hold, then the trivial solution of the stochastic differential equation (52) is not only uniformly stochastically asymptotically stable, but also uniformly stochastically asymptotically stable in the large.

Proof. Let $(x(t), y(t))$ be any solution of the stochastic differential equation (52). In view of (28) and estimate (29), the function $V(t, X)$ is positive definite. Furthermore, estimate (32) and inequality (33) show that the function $V(t, X)$ is radially unbounded and decrescent, respectively. It follows from (28), estimate (32), inequality (35), and the first inequality in (53) that all assumptions of Lemma 6 hold. Thus, by Lemma 6 the trivial solution of the stochastic differential equation (52) is uniformly stochastically asymptotically stable in the large. If estimate (32) is omitted then the trivial solution of the stochastic differential equation (52) is uniformly stochastically asymptotically stable. This completes the proof of Theorem 17.

Next, if the function $p\left(t, x, x^{\prime}\right)$ is replaced by $p(t) \epsilon$ $C\left(\mathbb{R}^{+}, \mathbb{R}^{+}\right)$, we have the following special case:

$$
\begin{aligned}
x^{\prime \prime}(t) & +g\left(x(t), x^{\prime}(t)\right) x^{\prime}(t)+f(x(t)) \\
& +\sigma x(t) \omega^{\prime}(t)=p(t),
\end{aligned}
$$

of (8). Equation (54) has the following equivalent system:

$$
\begin{aligned}
x^{\prime}(t)= & y(t), \\
y^{\prime}(t)= & p(t)-f(x)-g(x(t), y(t)) y(t) \\
& -\sigma x(t) \omega^{\prime}(t),
\end{aligned}
$$

with the following result.

Corollary 18. If assumptions (i) and (ii) of Theorem 12 hold and hypothesis (iii) is replaced by the boundedness of the function $p(t)$, then the solutions $(x(t), y(t))$ of the stochastic differential equation (55) are not only stochastically bounded but also uniformly stochastically bounded.

Proof. The proof of Corollary 18 is similar to the proof of Theorems 12 and 15 . This completes the proof of Corollary 18.

\section{Examples}

In this section we shall present two examples to illustrate the applications of the results we obtained in the previous section.

Example 1. Consider the second-order nonlinear nonautonomous stochastic differential equation

$$
\begin{aligned}
x^{\prime \prime} & +\left(3+\left|\cos \left(x x^{\prime}\right)\right|\right) x^{\prime}+x+\sin x+0.1 x \omega^{\prime}(t) \\
& =\left(1+2 t+\left|x x^{\prime}\right|\right)^{-1} .
\end{aligned}
$$


Equation (56) is equivalent to system

$$
\begin{aligned}
x^{\prime}= & y, \\
y^{\prime}= & (1+2 t+|x y|)^{-1}-(x+\sin x) \\
& -[3+|\cos (x y)|] y-0.1 x \omega^{\prime}(t) .
\end{aligned}
$$

Now from systems (9) and (57) we have the following relations:

(i) The function

$$
g(x, y):=3+|\cos (x y)|
$$

Noting that

$$
|\cos (x y)| \geq 0
$$

for all $x$ and $y$, it follows that

$$
g(x, y)=3+|\cos (x y)| \geq a=3,
$$

for all $x$ and $y$. The behaviour of the function $g(x, y)$ is shown below in Figure 1.

(ii) The function

$$
f(x):=x+\sin x
$$

Since

$$
-0.2 \leq F(x)=\frac{\sin x}{x} \leq 1
$$

for all $x \neq 0$, then we have

$$
1=b \leq \frac{f(x)}{x}=1+\frac{\sin x}{x} \leq B=2,
$$

for all $x \neq 0$ and since $\sigma:=0.1$ it follows that $\sigma^{2}<2 a b(b+1)^{-1}$ implies that $0<2.99$. The function $f(x) / x$ and its bounds are shown in Figure 2.

(iii) The function

$$
p(t, x, y):=\frac{1}{1+2 t+|x y|} .
$$

Clearly,

$$
|p(t, x, y)|=\frac{1}{1+2 t+|x y|} \leq 1=M_{0},
$$

for all $t \geq 0, x$, and $y$.

Now from items (i), (ii) above and (24), the continuously differentiable function $V(t, X)$ used for system (57) is

$$
2 V(t, X)=3 x^{2}+y^{2}+(3 x+y)^{2} .
$$

Different views of the function $V(t, X)$ are shown in Figure 3 . From (66), it is not difficult to show that

$$
\left(x^{2}+y^{2}\right) \leq V(t, X) \leq 3\left(x^{2}+y^{2}\right),
$$

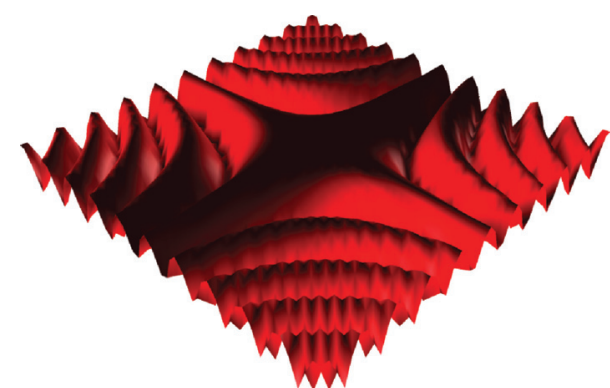

FIGURE 1: Behaviour of the function $g(x, y)$.

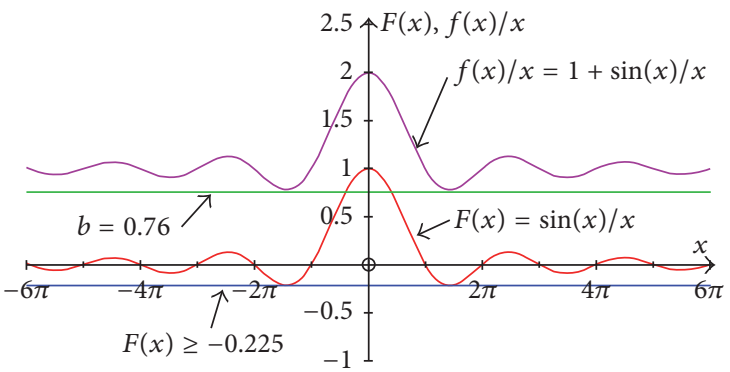

Figure 2: Bounds on the function $f(x) / x$.

for all $t \geq 0, x$, and $y$. From (35) and (67) we have $\delta_{0}=1$, $\delta_{1}=3, p=2$, and $q=2$, and thus, inequalities (67) satisfy condition (i) of Lemma 9. Also, from the first inequality in (67), we have

$$
V(t, X) \longrightarrow+\infty \quad \text { as } x^{2}+y^{2} \longrightarrow \infty .
$$

Estimate (68) verifies (32) (i.e., the function $V(t, X)$ defined by (66) is radially unbounded). Next, applying Itô's formula in (66) using system (57), we find that

$$
\begin{aligned}
L V(t, X)= & 12 x y+3 y^{2}-x(3 x+2 y)\left(1+\frac{\sin x}{x}\right) \\
& -y(3 x+2 y)(3+|\cos (x y)|)+\frac{1}{100} x^{2} \\
& -\frac{x}{10}(3 x+2 y) \\
& +(3 x+2 y)(1+2 t+|x y|)^{-1} .
\end{aligned}
$$

Using the estimates in items (i) to (iii) of Example 1 and the inequality $2 x_{1} x_{2} \leq x_{1}^{2}+x_{2}^{2}$ in (69), we obtain

$$
L V(t, X) \leq-2.9\left(x^{2}+y^{2}\right)+3(|x|+|y|),
$$

for all $t \geq 0, x$, and $y$. Inequality (70) satisfies inequality (40) where $\delta_{2}=2.9$ and $\delta_{3}=3$. Since

$$
(|x|-1.05)^{2}+(|y|-1.05)^{2} \geq 0,
$$

for all $x$ and $y$, it follows from inequality (70) that

$$
L V(t, X) \leq-1.45\left(x^{2}+y^{2}\right)+3.2,
$$




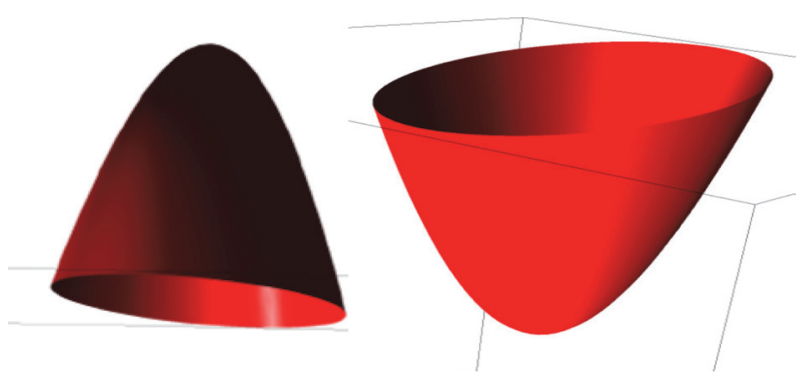

FIgUre 3: The behaviour of the function $V(t, X)$.

for all $t \geq 0, x$, and $y$. Inequality (72) satisfies assumption (ii) of Lemma 9 and estimate (44) with $\alpha(t)=\delta_{4}=1.45$ and $\beta(t)=\delta_{5}=3.2$. Since $r=p=q=2$, it follows that $\gamma=0$, so that assumption (iii) of Lemma 9 holds. In addition,

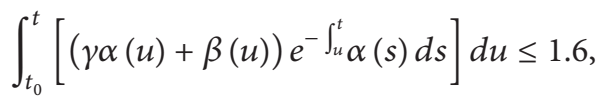

for all $t \geq t_{0} \geq 0$. Estimate (73) satisfies (23) and (45), with $M=2.6$. Furthermore,

$$
V_{x_{i}}(t, X) G_{i k}(t, X)=-\frac{1}{10}\left(3 x^{2}+2 x y\right),
$$

and

$$
\left|V_{x_{i}}(t, X) G_{i k}(t, X)\right| \leq \frac{2}{5}\left(x^{2}+y^{2}\right),
$$

for all $t \geq 0, x$, and $y$. Inequality (75) satisfies inequalities (20) and (21) with

$$
\lambda(t)=\frac{2}{5}\left(x^{2}+y^{2}\right) .
$$

Hence, by Corollary 11 (i), all solutions of stochastic differential equation (57) are uniformly stochastically bounded.

Example 2. If $p\left(t, x, x^{\prime}\right)=p(t, x, y)=0$ in (56) and system (57), we have the following stochastic differential equation:

$$
\begin{aligned}
x^{\prime \prime} & +\left(3+\left|\cos \left(x x^{\prime}\right)\right|\right) x^{\prime}+x+\sin x+0.1 x \omega^{\prime}(t) \\
& =0 .
\end{aligned}
$$

Equation (77) is equivalent to system

$$
\begin{aligned}
& x^{\prime}=y, \\
& y^{\prime}=-(x+\sin x)-[3+|\cos (x y)|] y-0.1 x \omega^{\prime}(t) .
\end{aligned}
$$

Now from systems (52) and (78) items (i) and (ii) of Example 1 hold. Also, equations (66), (67) and estimate (68) hold: that is,

$$
\begin{aligned}
& 2 V(t, X)=3 x^{2}+y^{2}+(3 x+y)^{2}, \\
& V(t, 0)=0, \forall t \geq 0 ; \\
& \left(x^{2}+y^{2}\right) \leq V(t, X) \leq 3\left(x^{2}+y^{2}\right) \quad \forall t \geq 0, x, y, \\
& V(t, X) \longrightarrow+\infty \quad \text { as } x^{2}+y^{2} \longrightarrow \infty .
\end{aligned}
$$

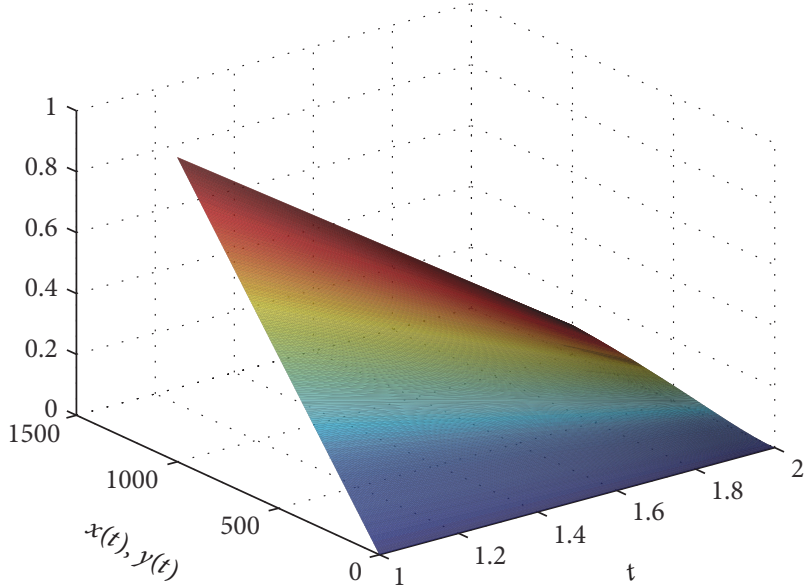

FIGURE 4: Graph of solutions of (56) in 3D.

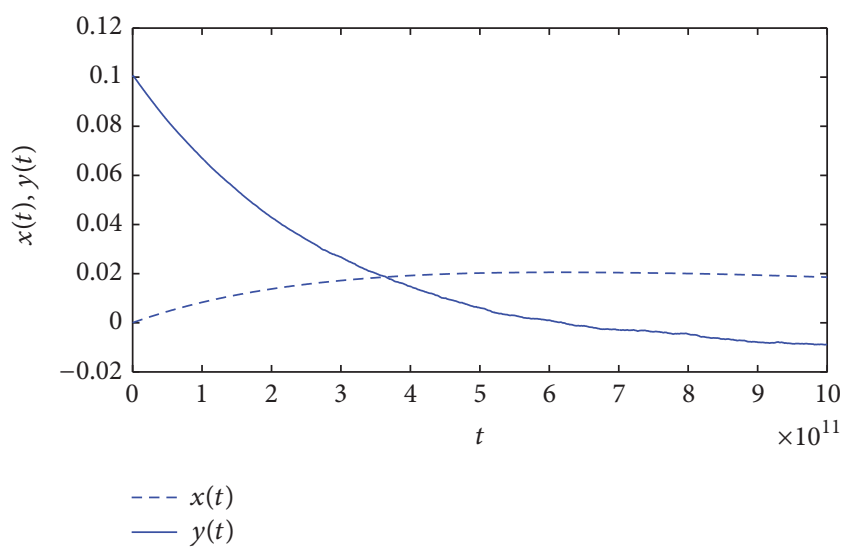

(a)

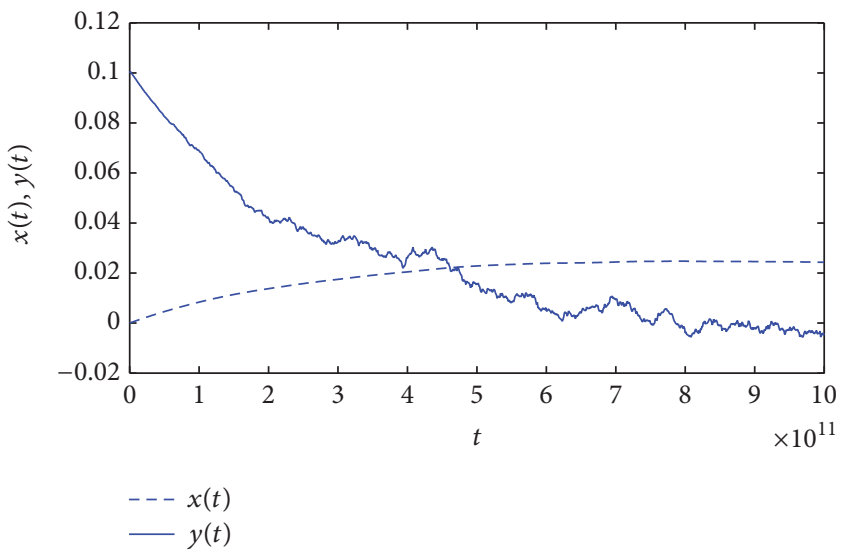

(b)

Figure 5

Furthermore, application of Itô's formula in (66) and using system (78) yield

$$
L V(t, X) \leq-2.9\left(x^{2}+y^{2}\right)
$$

for all $t \geq 0, x, y$ and thus

$$
L V(t, X) \leq 0,
$$




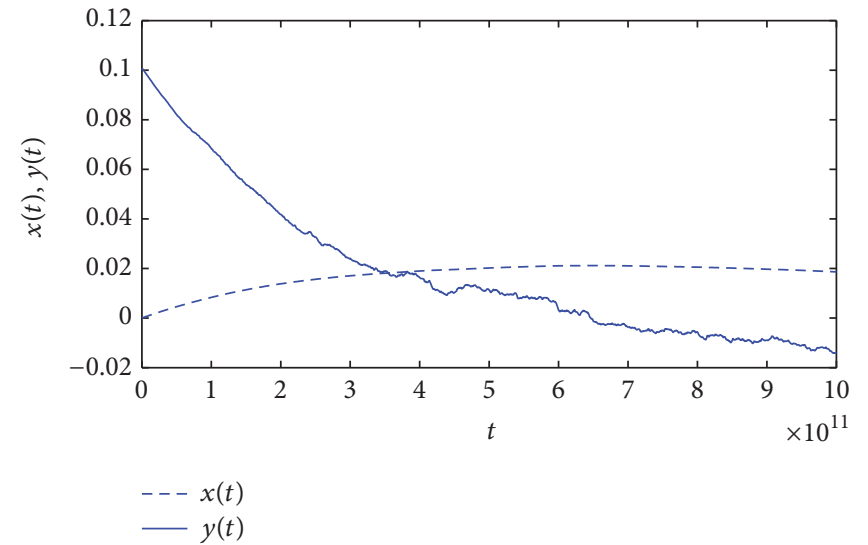

(a)

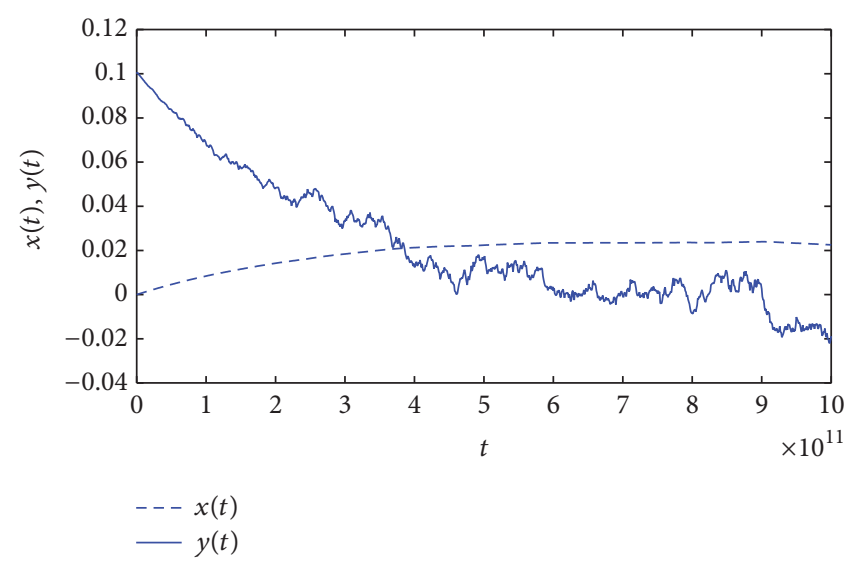

(b)

Figure 6

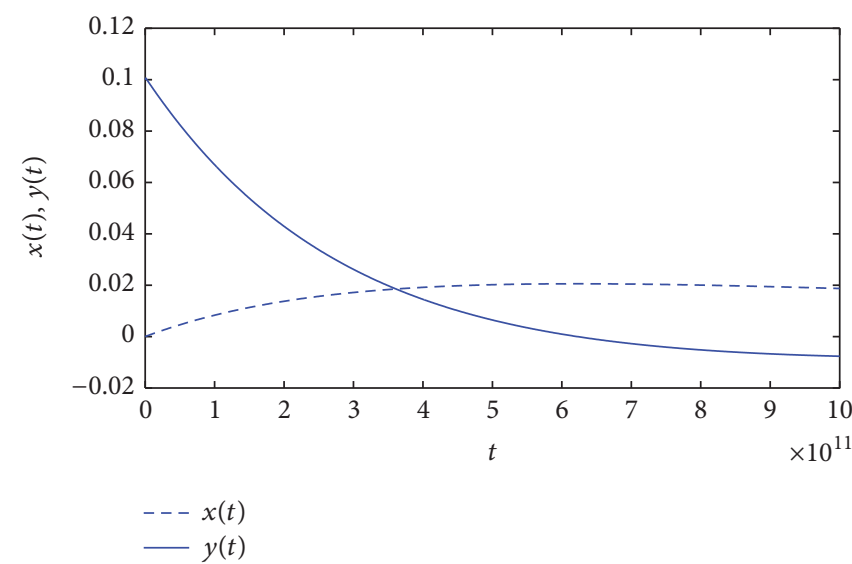

(a)

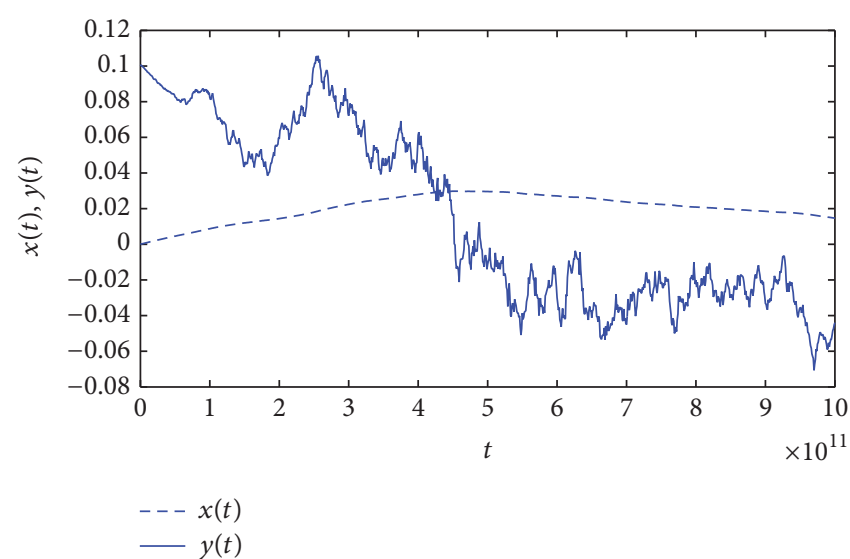

(b)

Figure 7

for all $t \geq 0, x$, and $y$. Moreover, from (79) and (80) all assumptions of Theorem 17 and Lemma 6 are satisfied. Thus, by Lemma 6 the trivial solution of system (78) is not only uniformly stochastically asymptotically stable but also uniformly stochastically asymptotically stable in the large. Finally, from (79) and (81) the function $V(t, X)$ is positive definite and

$$
L V(t, X) \leq 0, \quad \forall(t, X) \in \mathbb{R}^{+} \times \mathbb{R}^{2} .
$$

Hence, assumptions of Theorem 17 and Lemma 5 hold; by Theorem 17 and Lemma 5 the trivial solution of system (78) is stochastically stable.

Simulation of Solutions. In what follows, we shall now simulate the solutions of (56) (resp., system (57)) and (78) (resp., system (79)). Our approach depends on the EulerMaruyama method which enables us to get approximate numerical solution for the considered systems. It will be seen from our figures that the simulated solutions are bounded which justifies our given results. For instance, when $\sigma=0.1$, the numerical solutions of (56) in three-dimensional space are shown in Figure 4. If we vary the value of the noise in the numerical solution $(x(t), y(t))$ of system (57), as $\sigma=0.1$ and $\sigma=1.0$, we have Figures 5(a) and 5(b), respectively. It can be seen that, when the noise is increased, the stochasticity becomes more pronounced. The behaviour of the numerical solution $(x(t), y(t))$ of system (57) when $\sigma=0.5$ and $\sigma=2.0$ is shown in Figures 6(a) and 6(b), respectively. The behaviour of the numerical solution $(x(t), y(t))$ of system (57) for $\sigma=0$ and $\sigma=5.0$ is shown in Figures 7(a) and 7(b), respectively. For the case of (78), Figure 8 shows the closeness of the solution $(x(t))$ and the perturbed solution $\left(x_{\epsilon}(t)\right)$ for a very large $t$ which implies asymptotic stability in the large for the considered SDE.

\section{Competing Interests}

The authors declare that there are no competing interests regarding the publication of this paper. 


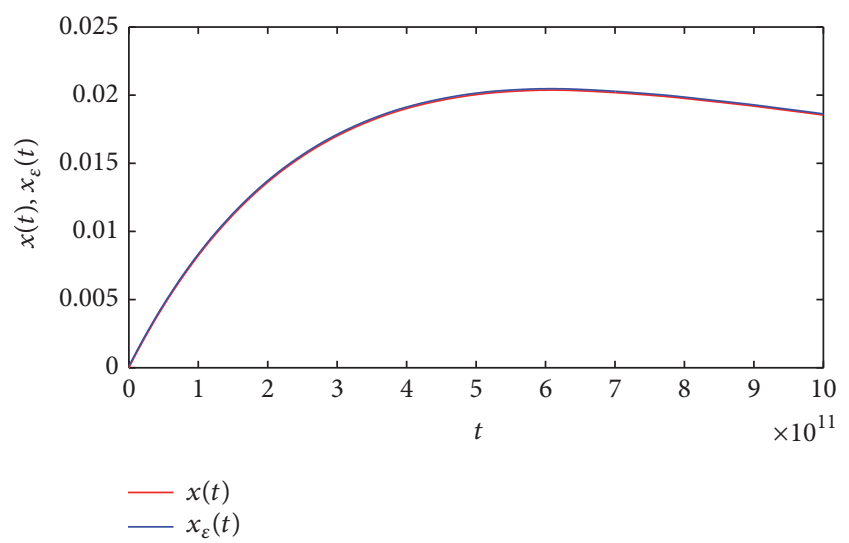

FIGURE 8: Graph of solutions of (78).

\section{References}

[1] L. Arnold, Stochastic Differential Equations: Theory and Applications, John Wiley \& Sons, 1974.

[2] T. A. Burton, Stability and Periodic Solutions of Ordinary and Functional Differential Equations, vol. 178 of Mathematics in Science and Engineering, Academic Press Inc., Orlando, Fla, USA, 1985.

[3] T. A. Burton, Volterra Integral and Differential Equations, Academic Press, New York, NY, USA, 1983.

[4] J. K. Hale, Theory of Functional Differential Equations, Springer, New York, NY, USA, 1977.

[5] B. Oksendal, Stochastic Differential Equations: An Introduction with Applications, Springer, 2000.

[6] L. Shaikihet, Lyapunov Functionals and Stability of Stochastic Functional Differential Equations, Springer International, 2013.

[7] T. Yoshizawa, Stability Theory and Existence of Periodic Solutions and almost Periodic Solutions, Spriger, New York, NY, USA, 1975.

[8] T. Yoshizawa, Stability Theory by Liapunov's Second Method, The Mathematical Society of Japan, 1966.

[9] A. M. A. Abou-El-Ela, A. I. Sadek, and A. M. Mahmoud, "On the stability of solutions for certain second-order stochastic delay differential equations," Differential Equations and Control Processes, no. 2, pp. 1-13, 2015.

[10] A. M. Abou-El-Ela, A. I. Sadek, A. M. Mahmoud, and R. O. Taie, "On the stochastic stability and boundedness of solutions for stochastic delay differential equation of the second order," Chinese Journal of Mathematics, vol. 2015, Article ID 358936, 8 pages, 2015.

[11] A. T. Ademola, Boundedness and Stability of Solutions to Certain Second Order Differential Equations, Differential Equations and Control Processes, 2015.

[12] A. T. Ademola, B. S. Ogundare, M. O. Ogundiran, and O. A. Adesina, "Periodicity, stability, and boundedness of solutions to certain second order delay differential equations," International Journal of Differential Equations, vol. 2016, Article ID 2843709, 10 pages, 2016.

[13] J. G. Alaba and B. S. Ogundare, "On stability and boundedness properties of solutions of certain second order nonautonomous nonlinear ordinary differential equation," Kragujevac Journal of Mathematics, vol. 39, no. 2, pp. 255-266, 2015.
[14] T. A. Burton and L. Hatvani, "Asymptotic stability of second order ordinary, functional, and partial differential equations," Journal of Mathematical Analysis and Applications, vol. 176, no. 1, pp. 261-281, 1993.

[15] B. Cahlon and D. Schmidt, "Stability criteria for certain secondorder delay differential equations with mixed coefficients," Journal of Computational and Applied Mathematics, vol. 170, no. 1, pp. 79-102, 2004.

[16] T. Caraballo, M. A. Diop, and A. S. Ndoye, "Fixed points and exponential stability for stochastic partial integro-differential equations with delays," Advances in Dynamical Systems and Applications, vol. 9, no. 2, pp. 133-147, 2014.

[17] A. Domoshnitsky, "Nonoscillation, maximum principles, and exponential stability of second order delay differential equations without damping term," Domoshnitsky Journal of Inequalities and Applications, vol. 2014, article 361, 2014.

[18] I. I. Gikhman and A. V. Skorokhod, Stochastische Differentialgleichungen, Akademie, Berlin, Germany, 1971 (Russian).

[19] I. I. Gikhman, On the Stability of the Solutions of Stochastic Differential Equations, Predel'nyye Teoremy i Statisticheskiye Vyvody, Tashkent, Uzbekistan, 1966.

[20] G. A. Grigoryan, "Boundedness and stability criteria for linear ordinary differential equations of the second order," Russian Mathematics, vol. 57, no. 12, pp. 8-15, 2013.

[21] A. F. Ivanov, Y. I. Kazmerchuk, and A. V. Swishchuk, "Theory, stochastic stability and applications of stochastic delay differential equations: a survey of recent results," in Differential Equations and Dynamical Systems, vol. 11, no. 1, 2003.

[22] F. Jedrzejewski and D. Brochard, "Lyapounv exponents and stability stochastic dynamical structures," 2000.

[23] Z. Jin and L. Zengrong, "On the global asymptotic behavior of solutions to a non autonomous generalized Liénard system," Journal of Mathematical Research and Exposition, vol. 21, no. 3, pp. 410-414, 2001.

[24] E. Kolarova, "An application of stochastic integral equations to electrical networks," Acta Electrotechnica et Informatica, vol. 8, no. 3, pp. 14-17, 2008.

[25] V. B. Kolmanovskii and L. E. Shaikhet, "A method for constructing Lyapunov functionals for stochastic systems with after effect," Differentsial'nye Uravneniya, vol. 29, no. 11, pp. 19092022, 1993.

[26] V. Kolmanovskii and L. Shaikhet, "Construction of Lyapunov functionals for stochastic hereditary systems: a survey of some recent results," Mathematical and Computer Modelling, vol. 36, no. 6, pp. 691-716, 2002.

[27] A. J. Kroopnick, "Bounded solutions to $x^{\prime \prime}+q(t) b(x)=f(t)$," International Journal of Mathematical Education in Science and Technology, vol. 41, no. 6, pp. 829-836, 2010.

[28] R. Liu and Y. Raffoul, "Boundedness and exponential stability of highly nonlinear stochastic differential equations," Electronic Journal of Differential Equations, vol. 2009, no. 143, pp. 1-10, 2009.

[29] X. Mao, "Some contributions to stochastic asymptotic stability and boundedness via multiple Lyapunov functions," Journal of Mathematical Analysis and Applications, vol. 260, no. 2, pp. 325340, 2001.

[30] B. S. Ogundare, A. T. Ademola, M. O. Ogundiran, and O. A. Adesina, "On the qualitative behaviour of solutions to certain second order nonlinear differential equation with delay," Annali dell'Universita' di Ferrara, 2016. 
[31] B. S. Ogundare and A. U. Afuwape, "Boundedness and stability properties of solutions of generalized Liénard equation," Kochi Journal of Mathematics, vol. 9, pp. 97-108, 2014.

[32] B. S. Ogundare and G. E. Okecha, "Boundedness, periodicity and stability of solutions to $\mathrm{x}^{\prime \prime}(\mathrm{t})+\mathrm{g}(\mathrm{x})+\mathrm{b}(\mathrm{t}) \mathrm{h}(\mathrm{x})=\mathrm{p}\left(\mathrm{t} ; \mathrm{x}, \mathrm{x}^{*}\right)$," Mathematical Sciences Research Journal, vol. 11, no. 5, pp. 432443, 2007.

[33] Y. N. Raffoul, "Boundedness and exponential asymptotic stability in dynamical systems with applications to nonlinear differential equations with unbounded terms," Advances in Dynamical Systems and Applications, vol. 2, no. 1, pp. 107-121, 2007.

[34] R. Rezaeyan and R. Farnoosh, "Stochastic differential equations and application of the Kalman-Bucy filter in the modeling of RC circuit," Applied Mathematical Sciences, vol. 4, no. 21-24, pp. 1119-1127, 2010.

[35] C. Tunç, "A note on the stability and boundedness of nonautonomous differential equations of second order with a variable deviating argument," Afrika Matematika, vol. 25, no. 2, pp. 417-425, 2014.

[36] C. Tunç, "A note on the bounded solutions to $x^{\prime \prime}+c\left(t, x, x^{\prime}\right)+$ $q(t) b(x)=f(t)$," Applied Mathematics \& Information Sciences, vol. 8, no. 1, pp. 393-399, 2014.

[37] C. Tunç, "Boundedness analysis for certain two-dimensional differential systems via a Lyapunov approach," Bulletin Mathematique de la Societe des Sciences Mathematiques de Roumanie, vol. 53, no. 1, pp. 61-68, 2010.

[38] C. Tunç, "New results on the existence of periodic solutions for rayleigh equation with state-dependent delay," Journal of Mathematical and Fundamental Sciences, vol. 45, no. 2, pp. 154162, 2013.

[39] C. Tunç, "Stability and boundedness in multi delay vector Liénard equation," Filomat, vol. 27, no. 3, pp. 435-445, 2013.

[40] C. Tunç, "Stability and boundedness of solutions of nonautonomous differential equations of second order," Journal of Computational Analysis and Applications, vol. 13, no. 6, pp. 1067-1074, 2011.

[41] C. Tunç, "Uniformly stability and boundedness of solutions of second order nonlinear delay differential equations," Applied and Computational Mathematics, vol. 10, no. 3, pp. 449-462, 2011.

[42] C. Tunç, "On the stability and boundedness of solutions of a class of nonautonomous differential equations of second order with multiple deviating arguments," Afrika Matematika, vol. 23, no. 2, pp. 249-259, 2012.

[43] C. Tunç and T. Ayhan, "Global existence and boundedness of solutions of a certain nonlinear integro-differential equation of second order with multiple deviating arguments," Journal of Inequalities and Applications, vol. 2016, article no. 46, 2016.

[44] F. Wang and H. Zhu, "Existence, uniqueness and stability of periodic solutions of a duffing equation under periodic and anti-periodic eigenvalues conditions," Taiwanese Journal of Mathematics, vol. 19, no. 5, pp. 1457-1468, 2015.

[45] Z. Xianfeng and J. Wei, "Stability and boundedness of a retarded Liénard-type equation," Chinese Quarterly Journal of Mathematics, vol. 18, no. 1, pp. 7-12, 2003.

[46] A. F. Yeniçerioğlu, "The behavior of solutions of second order delay differential equations," Journal of Mathematical Analysis and Applications, vol. 332, no. 2, pp. 1278-1290, 2007.

[47] A. F. Yeniçerioğlu, "Stability properties of second order delay integro-differential equations," Computers and Mathematics with Applications, vol. 56, no. 12, pp. 3109-3117, 2008.
[48] T. Yoshizawa, "Liapunov's function and boundedness of solutions," Funkcialaj Ekvacioj, vol. 2, pp. 71-103, 1958.

[49] W. Zhu, J. Huang, X. Ruan, and Z. Zhao, "Exponential stability of stochastic differential equation with mixed delay," Journal of Applied Mathematics, vol. 2014, Article ID 187037, 11 pages, 2014. 


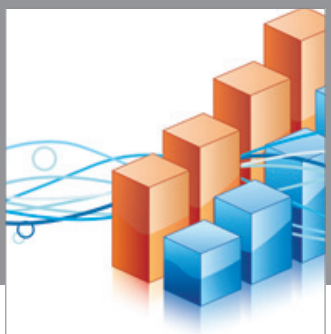

Advances in

Operations Research

vatem alat4

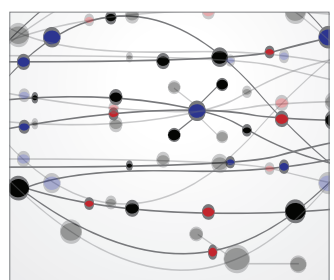

\section{The Scientific} World Journal
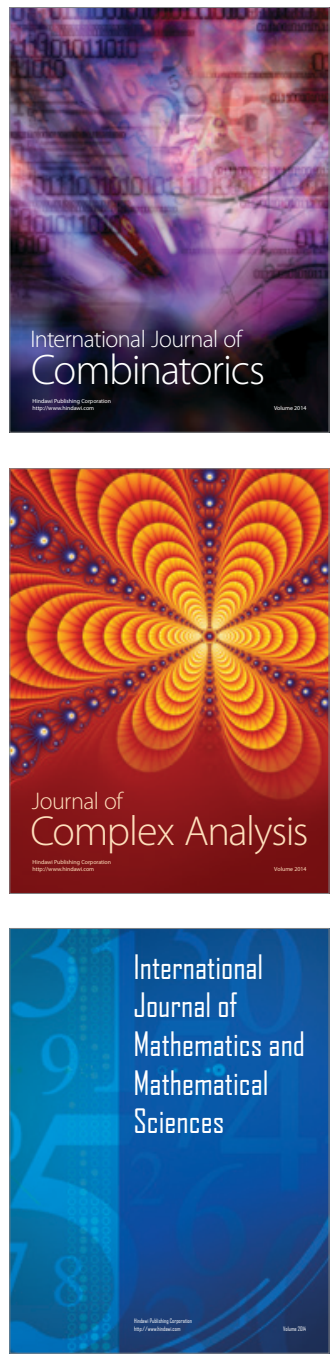
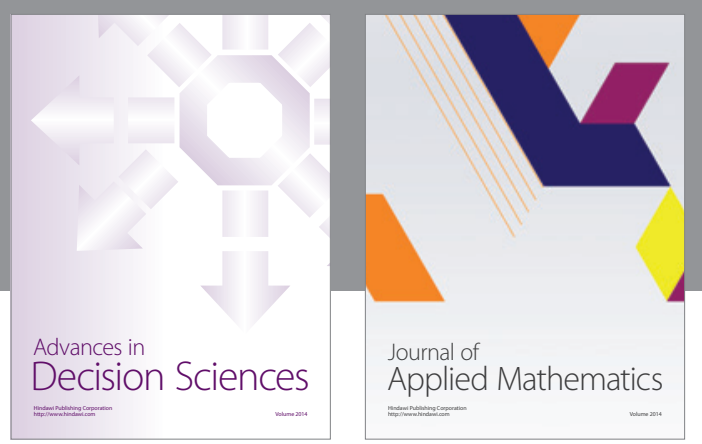

Algebra

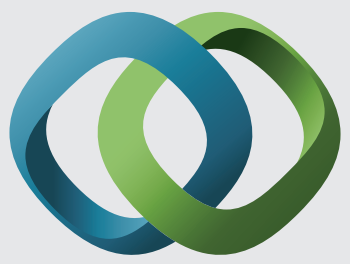

\section{Hindawi}

Submit your manuscripts at

http://www.hindawi.com
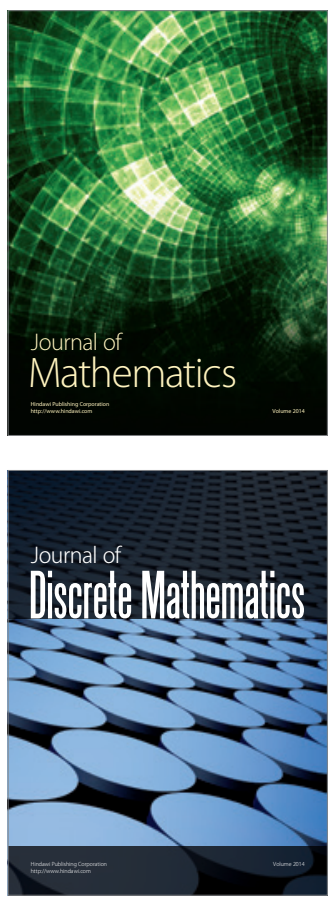

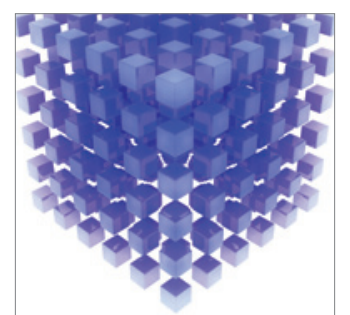

Mathematical Problems in Engineering
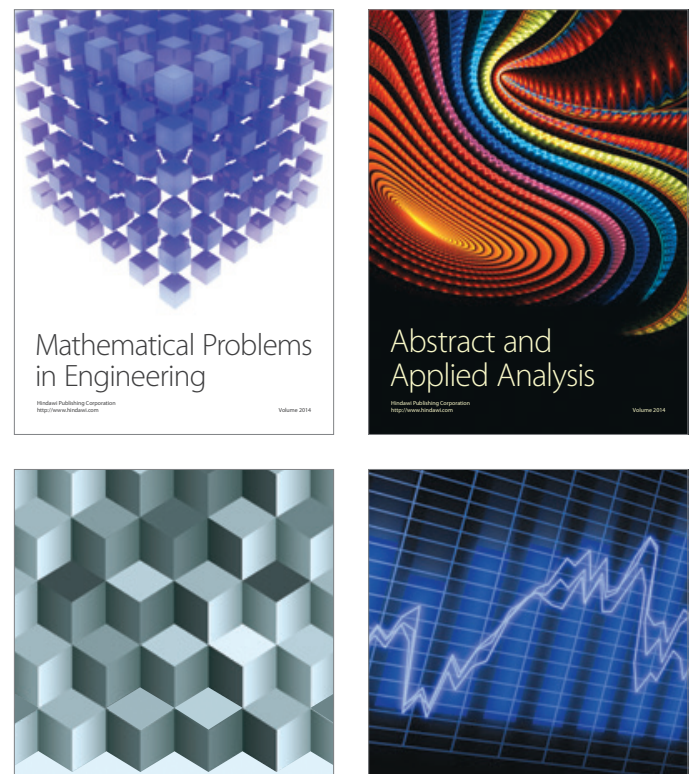

Journal of

Function Spaces

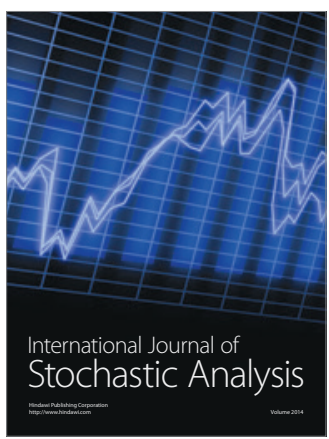

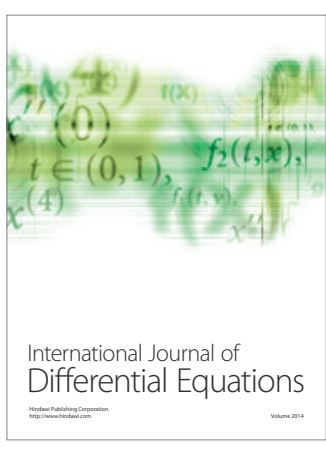
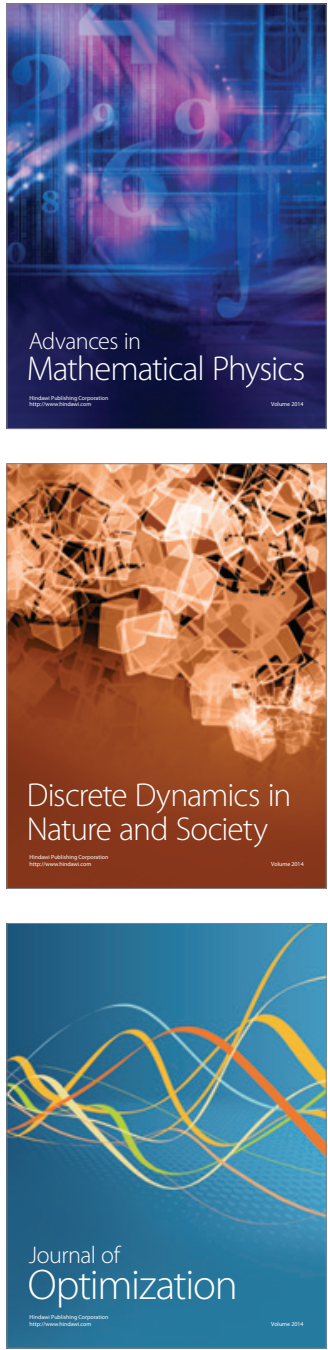